\title{
LEFSCHETZ PROPERTIES AND THE VERONESE CONSTRUCTION
}

\author{
Martina Kubitzke And Satoshi Murai
}

\begin{abstract}
In this paper, we investigate Lefschetz properties of Veronese subalgebras. We show that, for a sufficiently large $r$, the $r$ th Veronese subalgebra of a Cohen-Macaulay standard graded $K$-algebra has properties similar to the weak and strong Lefschetz properties, which we call the 'quasi-weak' and 'almost strong' Lefschetz properties. By using this result, we obtain new results on $h$ - and $g$-polynomials of Veronese subalgebras.
\end{abstract}

\section{Introduction}

Let $K$ be a field of characteristic 0 . For a standard graded (commutative) $K$-algebra $A=\bigoplus_{i>0} A_{i}$ and for an integer $r \geq 1$, the $K$-algebra $A^{\langle r\rangle}:=\bigoplus_{i>0} A_{i r}$, which is again standard graded, is called the $r$ th Veronese subalgebra of $A$. Quite recently, $h$-polynomials of Veronese subalgebras $[1,2,10]$ have been studied in different contexts. The focus of [2] lies on the analysis of the $h$-vector transformation and its asymptotics when passing from an algebra to its $r$ th Veronese subalgebra. More precisely, it is shown [2, Corollary 1.6] that if the $h$-polynomial of $A$ has non-negative integral coefficients, then, for sufficiently large $r$, the $h$-polynomial of $A^{\langle r\rangle}$ has only real zeros. In particular, this implies that the coefficient sequence of the $h$-polynomial of $A^{\langle r\rangle}$ is unimodal and log-concave. In [1], the asymptotic behavior of the $h$-vector transformation is worked out in greater detail and, in addition, an application to Ehrhart series is provided. Starting from the results in [2], it was proved in [10] that if the $h$-polynomial of $A$ has non-negative integral coefficients and if $r$ is larger than or equal to both the dimension of $A$ and the degree of the $h$-polynomial of $A$, then the $g$-polynomial of $A^{\langle r\rangle}$ is the $f$-polynomial of a simplicial complex and in particular its coefficient sequence is the Hilbert function of a standard graded $K$-algebra. Algebraically, the unimodality of the $h$-polynomial of a graded $K$-algebra is closely related to Lefschetz properties of Artinian graded $K$-algebras and in [10] the authors already raised the question of finding an algebraic proof of their results. This was the starting point for the work in this paper and one of its main purposes is to find a connection between Lefschetz properties and the Veronese construction. More precisely, we investigate Lefschetz properties of Veronese subalgebras of Cohen-Macaulay standard graded $K$-algebras and obtain new results on $h$ - and $g$-polynomials of Veronese subalgebras.

We recall some basics on Hilbert series and $h$-polynomials. The Hilbert series of a standard graded $K$-algebra $A=\bigoplus_{i \geq 0} A_{i}$ is the formal power series $\operatorname{Hilb}(A, t):=$ $\sum_{i \geq 0}\left(\operatorname{dim}_{K} A_{i}\right) t^{i}$. It is known that $\operatorname{Hilb}(A, t)$ is a rational function of the form

Received by the editors January 1, 2012. 
$\operatorname{Hilb}(A, t)=\left(h_{0}+h_{1} t+\cdots+h_{p} t^{p}\right) /(1-t)^{d}$, where each $h_{i}$ is an integer and where $d=\operatorname{dim} A$ is the Krull dimension of $A$ (see, e.g., [3, Section 4.1]). The polynomial

$$
h_{A}(t):=h_{0}+h_{1} t+\cdots+h_{p} t^{p}
$$

and the polynomial

$$
g_{A}(t):=h_{0}+\left(h_{1}-h_{0}\right) t+\cdots+\left(h_{\left\lfloor\frac{p}{2}\right\rfloor}-h_{\left\lfloor\frac{p}{2}\right\rfloor-1}\right) t^{\left\lfloor\frac{p}{2}\right\rfloor}
$$

are called the $h$-polynomial of $A$ and the g-polynomial of $A$, respectively. Here, $\lfloor x\rfloor$ denotes the integer part of $x$.

We first study the $k$-Lefschetz property and almost strong Lefschetz property, introduced in [9]. Let $A=\bigoplus_{i=0}^{p} A_{i}$ be a standard graded Artinian $K$-algebra, where $\operatorname{dim}_{K} A_{p}>0$. For an integer $k \geq 1$, we say that $A$ has the $k$-Lefschetz property if there is a linear form $w \in A_{1}$ such that the multiplication $w^{k-2 i}: A_{i} \rightarrow A_{k-i}: f \mapsto w^{k-2 i} f$ is injective for $0 \leq i \leq\left\lfloor\frac{k-1}{2}\right\rfloor$. The linear form $w$ is referred to as a $k$-Lefschetz element for $A$. If $A$ has the $(p-1)$-Lefschetz property, then we call it almost strong Lefschetz. An important consequence of the almost strong Lefschetz property is that if $A$ is almost strong Lefschetz, then the multiplication $w: A_{i} \rightarrow A_{i+1}$ is injective for $0 \leq i \leq\left\lfloor\frac{p}{2}\right\rfloor-1$ and the coefficient sequence of $g_{A}(t)$ becomes an $M$-sequence, namely, there is a standard graded Artinian $K$-algebra $B$ such that $g_{A}(t)=\operatorname{Hilb}(B, t)$. Indeed, it is easy to see that one can choose $B=A /\left(w A+\mathfrak{m}^{\left\lfloor\frac{p}{2}\right\rfloor+1}\right)$, where $\mathfrak{m}$ denotes the maximal homogeneous ideal of $A$.

We use Lefschetz properties to study $h$-polynomials of $A^{\langle r\rangle}$ in the following way. Let $A$ be a Cohen-Macaulay standard graded $K$-algebra of dimension $d$. A linear system of parameters (l.s.o.p. for short) for $A$ is a sequence $\Theta=\theta_{1}, \ldots, \theta_{d}$ of linear forms such that $\operatorname{dim}_{K} A / \Theta A<\infty$. Note that a l.s.o.p. for $A$ exists if $K$ is infinite, see, e.g., [12, p. 34]. For a l.s.o.p. $\Theta=\theta_{1}, \ldots, \theta_{d}$ for $A$ and for an integer $r \geq 1$, we write

$$
A_{\Theta}^{\langle r\rangle}:=A^{\langle r\rangle} /\left(\theta_{1}^{r} A^{\langle r\rangle}+\cdots+\theta_{d}^{r} A^{\langle r\rangle}\right) .
$$

We will see in Section 2, that $\theta_{1}^{r}, \ldots, \theta_{d}^{r}$ is a l.s.o.p. for $A^{\langle r\rangle}$. In particular, since $A^{\langle r\rangle}$ is Cohen-Macaulay (cf. [8, Chapter 3]), the Hilbert series of $A_{\Theta}^{\langle r\rangle}$ is equal to the $h$-polynomial of $A^{\langle r\rangle}$. As a consequence, the $h$-polynomial of $A^{\langle r\rangle}$ can be analyzed via Lefschetz properties for $A_{\Theta}^{\langle r\rangle}$. Our first result is the following.

Theorem 1.1. Let $A$ be a Cohen-Macaulay standard graded K-algebra of dimension $d$ and let $\Theta=\theta_{1}, \ldots, \theta_{d}$ be a l.s.o.p. for $A$. Let $r \geq 1$ be an integer and $s=\left\lfloor\frac{(r-1) d}{r}\right\rfloor$. Then $A_{\Theta}^{\langle r\rangle}$ has the s-Lefschetz property. Moreover, if $r \geq \operatorname{deg} h_{A}(t)$, then $A_{\Theta}^{\langle r\rangle}$ is almost strong Lefschetz.

In commutative algebra, the study of the weak Lefschetz property of Artinian graded $K$-algebras has shown to be of great interest. Recall, that a standard graded Artinian $K$-algebra $A=\bigoplus_{i=0}^{p} A_{i}$ is said to have the weak Lefschetz property if there is a linear form $w \in A_{1}$ such that the multiplication map $w: A_{i} \rightarrow A_{i+1}$ is either injective or surjective for all $i \geq 0$. Also, we say that $A$ is quasi-weak Lefschetz if there is a $1 \leq g<p$ and a linear form $w \in A_{1}$ such that the multiplication map $w: A_{i} \rightarrow A_{i+1}$ is injective for $0 \leq i \leq g-1$ and is surjective for $i \geq g+1$. Note that we do not set any condition on the multiplication map $w: A_{g} \rightarrow A_{g+1}$. If the multiplication map $w: A_{g} \rightarrow A_{g+1}$ is neither injective nor surjective, then the integer 
$g$ will be referred to as the $g a p$ of $A$ (w.r.t. $w$ ). We obtain the following result for the quasi-weak and the weak Lefschetz property.

Theorem 1.2. Let $A$ be a Cohen-Macaulay standard graded $K$-algebra of dimension $d$ and let $\Theta=\theta_{1}, \ldots, \theta_{d}$ be a l.s.o.p. for $A$.

(i) If $r \geq \operatorname{deg} h_{A}(t)$, then $A_{\Theta}^{\langle r\rangle}$ is quasi-weak Lefschetz.

(ii) If $d$ is even and $r \geq \max \left\{d, 2 \operatorname{deg} h_{A}(t)-d\right\}$, then $A_{\Theta}^{\langle r\rangle}$ has the weak Lefschetz property.

(iii) If $d$ is odd, $r \geq \frac{d}{2}$ and $\operatorname{deg} h_{A}(t) \leq \frac{d}{2}$, then $A_{\Theta}^{\langle r\rangle}$ has the weak Lefschetz property.

In Section 2, for the quasi-weak Lefschetz property, we will provide a result that is somewhat stronger, showing in particular that for $d \leq \operatorname{deg} h_{A}(t)$ a weaker assumption on $r$ is sufficient for guaranteeing the quasi-weak Lefschetz property.

We say that a polynomial $h_{0}+h_{1} t+\cdots+h_{p} t^{p} \in \mathbb{Z}_{>0}[t]$ is unimodal if there is a $1 \leq m \leq p$ such that $h_{0} \leq h_{1} \leq \cdots \leq h_{m} \geq h_{m+1} \geq \cdots \geq h_{p}$. Clearly, if a standard graded Artinian $K$-algebra $A$ is quasi-weak Lefschetz, then the $h$-polynomial of $A$ is unimodal. By combining Theorem 1.1 and Theorem 1.2 with some results on Gröbner basis for Veronese subalgebras due to Eisenbud, Reeves and Totaro [6], we also prove the following result on $h$-polynomials.

Theorem 1.3. Let $A$ be a Cohen-Macaulay standard graded $K$-algebra of dimension d. Let $r \geq 1$ be an integer and $s=\left\lfloor\frac{(r-1) d}{r}\right\rfloor$.

(i) If $r \geq \frac{1}{2}\left(\operatorname{deg} h_{A}(t)+1\right)$, then $h_{A\langle r\rangle}(t)$ is the $f$-polynomial of a flag simplicial complex.

(ii) If $r \geq \operatorname{deg} h_{A}(t)$, then $h_{A^{\langle r\rangle}}(t)$ is unimodal and $g_{A^{\langle r\rangle}}(t)$ is the $f$-polynomial of a simplicial complex.

(iii) Let $_{A_{A}^{\langle r\rangle}}(t)=\sum_{i \geq 0} h_{i}^{\langle r\rangle} t^{i}$. Then $h_{i}^{\langle r\rangle} \leq h_{s-i}^{\langle r\rangle}$ for all $i \leq\left\lfloor\frac{s-1}{2}\right\rfloor$.

\section{Lefschetz properties}

In this section, we study Lefschetz properties of $A_{\Theta}^{\langle r\rangle}$. In particular, we will provide the proofs of Theorem 1.1 and Theorem 1.2.

We start to fix some notation, which we will use throughout this section. In the following, we consider a Cohen-Macaulay standard graded $K$-algebra $A$ of dimension $d$ together with a l.s.o.p. $\Theta=\theta_{1}, \ldots, \theta_{d}$ for $A$. To prove Theorem 1.1 and Theorem 1.2, we use the following observation, which relates the Hilbert series of $A_{\Theta}^{\langle r\rangle}$ to the $h$-polynomial of the $r$ th Veronese subalgebra of $A$ : By Cohen-Macaulayness of $A, \Theta$ is not only a l.s.o.p. but also a regular sequence for $A$. Hence, $A$ is a finitely generated and free $K\left[\theta_{1}, \ldots, \theta_{d}\right]$-module. In particular, there exist homogeneous elements $u_{1}, \ldots, u_{m}$ of $A$ such that we have the decomposition

$$
A=\bigoplus_{j=1}^{m} u_{j} \cdot K\left[\theta_{1}, \ldots, \theta_{d}\right]
$$

as $K\left[\theta_{1}, \ldots, \theta_{d}\right]$-modules (see, e.g., [12, Chapter 1$]$ ). Note that $u_{1}, \ldots, u_{m}$ is a $K$-basis of $A / \Theta A$. Moreover, since the Hilbert series of $A / \Theta A$ is equal to the $h$-polynomial of 
$A$ (cf., [3, Remark 4.1.11]), we have

$$
\operatorname{deg} u_{j} \leq \operatorname{deg} h_{A}(t)
$$

for all $1 \leq j \leq m$. Let $r \geq 1$ be an integer. We will show that $\theta_{1}^{r}, \ldots, \theta_{d}^{r}$ is a l.s.o.p. for $A^{\langle r\rangle}$. We include this proof since we could not find any reference to this fact in the literature. From (2.1) we infer that the $r$ th Veronese subalgebra $A^{\langle r\rangle}$ decomposes as

$$
A^{\langle r\rangle}=\bigoplus_{j=1}^{m} u_{j} \cdot\left(\bigoplus_{i \geq 0} K\left[\theta_{1}, \ldots, \theta_{d}\right]_{i r-\operatorname{deg} u_{j}}\right),
$$

where we set $K\left[\theta_{1}, \ldots, \theta_{d}\right]_{k}:=\{0\}$ if $k<0$.

And for the quotient $A_{\Theta}^{\langle r\rangle}=A^{\langle r\rangle} /\left(\theta_{1}^{r} A^{\langle r\rangle}+\cdots+\theta_{d}^{r} A^{\langle r\rangle}\right)$, we obtain

$$
A_{\Theta}^{\langle r\rangle}=\bigoplus_{j=1}^{m} u_{j} \cdot\left(\bigoplus_{i \geq 0}\left(K\left[\theta_{1}, \ldots, \theta_{d}\right] /\left(\theta_{1}^{r}, \ldots, \theta_{d}^{r}\right)\right)_{i r-\operatorname{deg} u_{j}}\right)=\left(A /\left(\theta_{1}^{r}, \ldots, \theta_{d}^{r}\right)\right)^{\langle r\rangle}
$$

as $K\left[\theta_{1}, \ldots, \theta_{d}\right]^{\langle r\rangle}$-modules. Being the grading of $A_{\Theta}^{\langle r\rangle}$ induced by the usual $\mathbb{Z}$-grading of $K\left[\theta_{1}, \ldots, \theta_{d}\right]$, we know that the homogeneous component $\left(A_{\Theta}^{\langle r\rangle}\right)_{i}$ of $A_{\Theta}^{\langle r\rangle}$ of degree $i$ is given by

$$
\left(A_{\Theta}^{\langle r\rangle}\right)_{i}=\bigoplus_{j=1}^{m} u_{j} \cdot\left(K\left[\theta_{1}, \ldots, \theta_{d}\right] /\left(\theta_{1}^{r}, \ldots, \theta_{d}^{r}\right)\right)_{i r-\operatorname{deg} u_{j}} .
$$

Moreover, since $A$ is integral over $A^{\langle r\rangle}$, both algebras have the same Krull dimension (see [8, Chapter 3] and [4, Proposition 3.3]). Since the right-hand side of (2.2) has finite length, we conclude that $\theta_{1}^{r}, \ldots, \theta_{d}^{r}$ is a l.s.o.p. for $A^{\langle r\rangle}$. This together with the fact that the Cohen-Macaulay property is preserved under taking Veronese subalgebras (cf. [8, Chapter 3]) implies that the Hilbert series of $A_{\Theta}^{\langle r\rangle}$ equals the $h$-polynomial of $A^{\langle r\rangle}$. In particular, since $\max \left\{\operatorname{deg} u_{j} \mid 1 \leq j \leq m\right\}=\operatorname{deg} h_{A}(t)$ and since the maximum degree in $K\left[\theta_{1}, \ldots, \theta_{d}\right] /\left(\theta_{1}^{r}, \ldots, \theta_{d}^{r}\right)$ is $(r-1) d$, it follows directly from $(2.3)$ that

$$
\operatorname{deg} h_{A^{\langle r\rangle}}(t)=\left\lfloor\frac{d(r-1)+\operatorname{deg} h_{A}(t)}{r}\right\rfloor .
$$

Note that the above equation (2.4) holds for any standard graded $K$-algebra $A$ whose $h$-polynomial has non-negative coefficients (e.g., use [2, Corollary 1.2]).

For the proof of Theorem 1.1 and Theorem 1.2, we need the following fact proved by Stanley [11] and Watanabe [14].

Lemma 2.1. Let $K$ be a field of characteristic 0 and let $r \geq 1$ be an integer. For integers $0 \leq i<j$, the multiplication map

$$
\begin{aligned}
\times\left(x_{1}+\cdots+x_{d}\right)^{j-i}:\left(K\left[x_{1}, \ldots, x_{d}\right] /\left(x_{1}^{r}, \ldots, x_{d}^{r}\right)\right)_{i} & \rightarrow\left(K\left[x_{1}, \ldots, x_{d}\right] /\left(x_{1}^{r}, \ldots, x_{d}^{r}\right)\right)_{j} \\
p & \mapsto\left(x_{1}+\cdots+x_{d}\right)^{j-i} \cdot p
\end{aligned}
$$

is injective if $i+j \leq(r-1) d$ and is surjective if $i+j \geq(r-1) d$. 
We have now laid the necessary foundations for giving the proof of Theorem 1.1.

Proof of Theorem 1.1. Let $w=\left(\theta_{1}+\cdots+\theta_{d}\right)^{r}$. We prove that $w$ is an $s$-Lefschetz element of $A_{\Theta}^{\langle r\rangle}$, namely, we will show that the multiplication

$$
\times w^{s-2 i}:\left(A_{\Theta}^{\langle r\rangle}\right)_{i} \rightarrow\left(A_{\Theta}^{\langle r\rangle}\right)_{s-i}
$$

is injective for $0 \leq i \leq\left\lfloor\frac{s-1}{2}\right\rfloor$. By decomposition (2.2), it is enough to prove that, for $1 \leq j \leq m$, the multiplication

$$
\begin{gathered}
\times w^{s-2 i}:\left(K\left[\theta_{1}, \ldots, \theta_{d}\right] /\left(\theta_{1}^{r}, \ldots, \theta_{d}^{r}\right)\right)_{i r-\operatorname{deg} u_{j}} \\
\rightarrow\left(K\left[\theta_{1}, \ldots, \theta_{d}\right] /\left(\theta_{1}^{r}, \ldots, \theta_{d}^{r}\right)\right)_{(s-i) r-\operatorname{deg} u_{j}}
\end{gathered}
$$

is injective for $0 \leq i \leq\left\lfloor\frac{s-1}{2}\right\rfloor$. The desired injectivity follows from Lemma 2.1 since $i r-\operatorname{deg} u_{j}+(s-i) r-\operatorname{deg} u_{j} \leq s r \leq(r-1) d$.

Finally, if $r \geq \operatorname{deg} h_{A}(t)$, then $\operatorname{deg} \operatorname{Hilb}\left(A_{\Theta}^{\langle r\rangle}, t\right)=\operatorname{deg} h_{A^{\langle r\rangle}}(t) \leq s+1$ by (2.4), which implies that $A_{\Theta}^{\langle r\rangle}$ is almost strong Lefschetz.

We now proceed to the proof of Theorem 1.2. Part (i), i.e., the statement concerning the quasi-weak Lefschetz property, follows from the following stronger result.

Theorem 2.2. Let $A$ be a Cohen-Macaulay standard graded $K$-algebra of dimension $d$ and let $\Theta=\theta_{1}, \ldots, \theta_{d}$ be a l.s.o.p. for $A$. Then $A_{\Theta}^{\langle r\rangle}$ is quasi-weak Lefschetz if

(a) $d$ is even and one of the following conditions holds:

(i) $d \leq \frac{1}{2} \operatorname{deg} h_{A}(t)$ and $r \geq \frac{2 \operatorname{deg} h_{A}(t)-d}{3}$,

(ii) $\frac{1}{2} \operatorname{deg} h_{A}(t) \leq d \leq \operatorname{deg} h_{A}(t)$ and $r \geq d$,

(iii) $\operatorname{deg} h_{A}(t) \leq d \leq \frac{3}{2} \operatorname{deg} h_{A}(t)$ and $r \geq 2 \operatorname{deg} h_{A}(t)-d$,

(iv) $\frac{3}{2} \operatorname{deg} h_{A}(t) \leq d \leq 3 \operatorname{deg} h_{A}(t)$ and $r \geq \frac{d}{3}$,

(v) $d \geq 3 \operatorname{deg} h_{A}(t)$ and $r \geq \operatorname{deg} h_{A}(t)$,or,

(b) $d$ is odd and one of the following conditions holds:

(i) $d \leq \operatorname{deg} h_{A}(t)$ and $r \geq \operatorname{deg} h_{A}(t)-\frac{d}{2}$,

(ii) $\operatorname{deg} h_{A}(t) \leq d \leq 2 \operatorname{deg} h_{A}(t)$ and $r \geq \frac{d}{2}$,

(iii) $d \geq 2 \operatorname{deg} h_{A}(t)$ and $r \geq \operatorname{deg} h_{A}(t)$.

Proof. Before providing the proofs for each set of conditions separately, we start with a general discussion that can be used in all cases. Let $w:=\left(\theta_{1}+\cdots+\theta_{d}\right)^{r}$. Our aim is to show that in all parts of the theorem, $w$ is a quasi-weak Lefschetz element for $A_{\Theta}^{\langle r\rangle}$. Using the same notations as at the beginning of this section, we know from (2.2) that as $K\left[\theta_{1}, \ldots, \theta_{d}\right]^{\langle r\rangle}$-modules, we have the decomposition:

$$
A_{\Theta}^{\langle r\rangle}=\bigoplus_{j=1}^{m} u_{j}\left(\bigoplus_{i \geq 0}\left(K\left[\theta_{1}, \ldots, \theta_{d}\right] /\left(\theta_{1}^{r}, \ldots, \theta_{d}^{r}\right)\right)_{i r-\operatorname{deg} u_{j}}\right) .
$$

Thus, in order to show that the multiplication

$$
\times w:\left(A_{\Theta}^{\langle r\rangle}\right)_{i} \rightarrow\left(A_{\Theta}^{\langle r\rangle}\right)_{i+1}
$$


is injective and surjective for a certain $i \geq 0$, it suffices to show that for all $1 \leq j \leq m$ the multiplication

$$
\times w:\left(K\left[\theta_{1}, \ldots, \theta_{d}\right] /\left(\theta_{1}^{r}, \ldots, \theta_{d}^{r}\right)\right)_{i r-\operatorname{deg} u_{j}} \rightarrow\left(K\left[\theta_{1}, \ldots, \theta_{d}\right] /\left(\theta_{1}^{r}, \ldots, \theta_{d}^{r}\right)\right)_{(i+1) r-\operatorname{deg} u_{j}}
$$

is injective and surjective, respectively, for the same $i$.

We first consider case (a) (i). Suppose that $d$ is even, $d \leq \frac{1}{2} \operatorname{deg} h_{A}(t)$ and $r \geq$ $\frac{2 \operatorname{deg} h_{A}(t)-d}{3}$. Combining the latter two conditions in particular yields $r \geq d$. Our aim is to use Lemma 2.1. We first show that the multiplication in (2.5) is injective for $0 \leq i \leq \frac{d}{2}-1$ and for all $1 \leq j \leq m$. For all $1 \leq j \leq m$ it holds that

$$
2 i r+r-2 \operatorname{deg} u_{j} \leq d r-r \leq(r-1) d+d-r \leq(r-1) d,
$$

where the first and the last inequality follow from $\operatorname{deg} u_{j} \geq 0$ for $1 \leq j \leq m$ and $r \geq d$, respectively. Hence, Lemma 2.1 implies the desired injectivity.

Next, we show that the multiplication in (2.5) is surjective for $i \geq \frac{d}{2}+1$. As in the previous case, for $1 \leq j \leq m$, we compute

$$
2 i r+r-2 \operatorname{deg} u_{j} \geq d r+3 r-2 \operatorname{deg} h_{A}(t) \geq(r-1) d,
$$

where for the first inequality we use that $\operatorname{deg} u_{j} \leq \operatorname{deg} h_{A}(t)$ for $1 \leq j \leq m$, and the last inequality holds since $r \geq \frac{2 \operatorname{deg} h_{A}(t)-d}{3}$. Surjectivity now follows from Lemma 2.1.

The cases (a) (ii)-(iv) and (b) (i)-(ii) follow from almost literally the same arguments, taking into account the different ranges and bounds for $d$ and $r$, respectively, as well as the different location of the gap. Indeed, if there is a gap, then it is at position $\frac{d}{2}$ in the cases (a) (i)-(ii), and at position $\frac{d}{2}-1$ in the cases (iii)-(iv). In the situation of (b) (i)-(ii), the gap — if existing — lies at position $\frac{d-1}{2}$.

The cases (a) (v) and (b) (iii) have to be treated slightly differently. Let $s=$ $\left\lfloor\frac{(r-1) d}{r}\right\rfloor$. By an analogous reasoning as for the other cases one infers that the multiplication in (2.5) is surjective for $i \geq \frac{s}{2}+1$. On the other hand, Theorem 1.1 says that $A_{\Theta}^{\langle r\rangle}$ is $s$-Lefschetz. In particular, the multiplication map in (2.5) is injective for $i \leq \frac{s-1}{2}$. Hence, we conclude that $A_{\Theta}^{\langle r\rangle}$ is quasi-weak Lefschetz with a possible gap at position $\left\lfloor\frac{s+1}{2}\right\rfloor$.

Remark 2.3. We want to remark that the arguments in the above proof do only depend on the effective size of $r$ and not on the precise relation between $d$ and $\operatorname{deg} h_{A}(t)$. Moreover, the proofs of (a) (iv) and (b) (iii) do not use the fact that $d \leq 3 \operatorname{deg} h_{A}(t)$ and $d \leq 2 \operatorname{deg} h_{A}(t)$, respectively. We only include these restrictions since for $d>\operatorname{deg} h_{A}(t)$ part (a) (v) and part (b) (iii) provide better, i.e., smaller bounds for $r$. In particular, this allows us to conclude, that if $d$ is even, the gap if existing - is at position $\frac{d}{2}$ if $r \geq \max \left\{d, \frac{2 \operatorname{deg} h_{A}(t)-d}{3}\right\}$ and at position $\frac{d}{2}+1$ if $r \geq \max \left\{\frac{d}{3}, 2 \operatorname{deg} h_{A}(t)-d\right\}$. If $d$ is odd and $r \geq \max \left\{\frac{d}{2}, \operatorname{deg} h_{A}(t)-\frac{d}{2}\right\}$, the gap is at position $\frac{d-1}{2}$. This will be relevant for the proof of Theorem 1.2 (ii) and (iii).

Proof of Theorem 1.2. Part (i) can readily be deduced from Theorem 2.2. To show part (ii), note that - independent of $d$ - it follows from Theorem 2.2 (a) (i)-(iv) and Remark 2.3 that $A_{\Theta}^{\langle r\rangle}$ is quasi-weak Lefschetz. Since there can exist at most one gap, we infer from Remark 2.3 that $A_{\Theta}^{\langle r\rangle}$ is indeed weak Lefschetz. 
For part (iii), let $r \geq \max \left\{\frac{d}{2}, \operatorname{deg} h_{A}(t)-\frac{d}{2}\right\}$. By Theorem 2.2 and Remark 2.3, we know that $A_{\Theta}^{\langle r\rangle}$ is quasi-weak Lefschetz with a possible gap being at position $\frac{d-1}{2}$. Assume, in addition, that $\operatorname{deg} h_{A}(t) \leq \frac{d}{2}$. We claim that the multiplication map

$$
\times w:\left(A_{\Theta}^{\langle r\rangle}\right)_{\frac{d-1}{2}} \rightarrow\left(A_{\Theta}^{\langle r\rangle}\right)_{\frac{d-1}{2}+1}
$$

is surjective. The desired surjectivity follows from Lemma 2.1 since for all $1 \leq j \leq m$ it holds that

$$
r\left(\frac{d-1}{2}+\frac{d-1}{2}+1\right)-2 \operatorname{deg} u_{j} \geq r d-2 \operatorname{deg} h_{A}(t) \geq(r-1) d .
$$

We conclude that $A_{\Theta}^{\langle r\rangle}$ has the weak Lefschetz property.

Remark 2.4. Theorem 1.2 (ii) says that, for any even dimensional Cohen-Macaulay graded $K$-algebra $A$, the algebra $A_{\Theta}^{\langle r\rangle}$ has the weak Lefschetz property for $r \gg 0$. Unfortunately, this fact does not hold for odd dimensional Cohen-Macaulay graded $K$-algebras. Let $A=K\left[x_{1}, \ldots, x_{8}\right] /\left(\left(x_{1}^{2}, x_{1} x_{2}, x_{1} x_{3}, x_{1} x_{4}, x_{1} x_{5}\right)+\left(x_{2}, x_{3}, x_{4}, x_{5}\right)^{3}\right)$. Then $A$ is a Cohen-Macaulay graded $K$-algebra of dimension 3 with the $h$-polynomial $h_{A}(t)=1+5 t+10 t^{2}$ and $\Theta=x_{6}, x_{7}, x_{8}$ is a l.s.o.p. for $A$, but $A_{\Theta}^{\langle r\rangle}$ does not have the weak Lefschetz property for any $r \geq 3$.

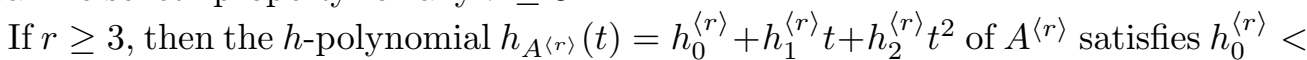
$h_{1}^{\langle r\rangle}<h_{2}^{\langle r\rangle}$. However, there are no linear forms $w$ such that $\times w:\left(A_{\Theta}^{\langle r\rangle}\right)_{1} \rightarrow\left(A_{\Theta}^{\langle r\rangle}\right)_{2}$ is injective. Consider the $K$-vector spaces $V=x_{1}\left(K\left[x_{6}, x_{7}, x_{8}\right] /\left(x_{6}^{r}, x_{7}^{r}, x_{8}^{r}\right)\right)_{r-1} \subset$ $\left(A_{\Theta}^{\langle r\rangle}\right)_{1}$ and $W=x_{1}\left(K\left[x_{6}, x_{7}, x_{8}\right] /\left(x_{6}^{r}, x_{7}^{r}, x_{8}^{r}\right)\right)_{2 r-1} \subset\left(A_{\Theta}^{\langle r\rangle}\right)_{2}$. Then, for any linear form $w \in A_{\Theta}^{\langle r\rangle}$ we have $w V \subset W$, since $x_{1} x_{i}=0$ in $A$ for $i=1,2, \ldots, 5$, but

$$
\begin{aligned}
\operatorname{dim}_{K} V & =\operatorname{dim}_{K}\left(K\left[x_{6}, x_{7}, x_{8}\right] /\left(x_{6}^{r}, x_{7}^{r}, x_{8}^{r}\right)\right)_{r-1} \\
& >\operatorname{dim}_{K}\left(K\left[x_{6}, x_{7}, x_{8}\right] /\left(x_{6}^{r}, x_{7}^{r}, x_{8}^{r}\right)\right)_{2 r-1}=\operatorname{dim}_{K} W,
\end{aligned}
$$

where the inequality follows since $\operatorname{dim}_{K}\left(K\left[x_{6}, x_{7}, x_{8}\right] /\left(x_{6}^{r}, x_{7}^{r}, x_{8}^{r}\right)\right)_{r-1}=\left(\begin{array}{c}r+1 \\ r-1\end{array}\right)$ and $\operatorname{dim}_{K}\left(K\left[x_{6}, x_{7}, x_{8}\right] /\left(x_{6}^{r}, x_{7}^{r}, x_{8}^{r}\right)\right)_{2 r-1}=\operatorname{dim}_{K}\left(K\left[x_{6}, x_{7}, x_{8}\right] /\left(x_{6}^{r}, x_{7}^{r}, x_{8}^{r}\right)\right)_{r-2}=\left(\begin{array}{c}r \\ r-2\end{array}\right)$. This fact implies that the multiplication $\times w: V \rightarrow W$ is not injective.

\section{Consequences on $h$-vectors}

In this section, we prove Theorem 1.3. Throughout this section, we let $S=K\left[x_{1}, \ldots\right.$, $x_{n}$ ] be a standard graded polynomial ring over a field $K$. For an integer $r \geq 1$, let

$$
T_{\langle r\rangle}=K\left[z_{m}: m \text { is a monomial in } S \text { of degree } r\right] \text {, }
$$

where each $z_{m}$ is a variable. Then there is a ring homomorphism

$$
\begin{aligned}
\phi_{r}: T_{\langle r\rangle} & \longrightarrow S^{\langle r\rangle} \\
z_{m} & \mapsto m
\end{aligned}
$$

For a homogeneous ideal $I \subset S$, let $I^{\langle r\rangle}:=\bigoplus_{j \geq 0} I_{j r}$. Then $I^{\langle r\rangle}$ is a graded ideal of $S^{\langle r\rangle}$ and $(S / I)^{\langle r\rangle}=S^{\langle r\rangle} / I^{\langle r\rangle}$. Also, the ring $(S / I)^{\langle r\rangle}$ is isomorphic to $T_{\langle r\rangle} / \phi_{r}^{-1}\left(I^{\langle r\rangle}\right)$.

To prove the main result, we need a few known results on Gröbner bases of $\phi^{-1}\left(I^{\langle r\rangle}\right)$ proved by Eisenbud et al. [6]. We refer the readers to [5] for the basics on Gröbner basis theory. 
Let $>_{\text {rev }}$ be the reverse lexicographic order on $S$ induced by $x_{1}>\cdots>x_{n}$, and let $\succ_{\text {rev }}$ be the reverse lexicographic order on $T_{\langle r\rangle}$ such that the ordering of the variables is defined by $z_{m} \succ_{\text {rev }} z_{m^{\prime}}$ if $m>_{\text {rev }} m^{\prime}$. For a monomial $m \in S$, we write $\max (m)$ (resp. $\min (m))$ for the largest (resp. smallest) integer $i$ such that $x_{i}$ divides $m$. We say that a monomial

$$
u=z_{m_{1}} z_{m_{2}} \cdots z_{m_{k}} \in T_{\langle r\rangle},
$$

where $m_{1}>_{\text {rev }} \cdots>_{\text {rev }} m_{k}$, is standard if $\max \left(m_{i}\right) \leq \min \left(m_{i+1}\right)$ for $1 \leq i \leq k-1$. The following fact was shown in the proof of [6, Proposition 6]. See also [13, Theorem $14.2]$.

Lemma 3.1. A monomial $u \in T_{\langle r\rangle}$ is standard if and only if $u \notin \mathrm{in}_{\succ_{\mathrm{rev}}}\left(\operatorname{ker} \phi_{r}\right)$.

The above lemma implies the next result.

Lemma 3.2. Let $r \geq 1$ and $1 \leq \ell \leq n$ be integers. Let $I \subset S$ be a monomial ideal and $J=I+\left(x_{n}^{r}, x_{n-1}^{r}, \ldots, x_{\ell}^{r}\right)$. Then

$$
\operatorname{in}_{\succ_{\mathrm{rev}}} \phi_{r}^{-1}\left(J^{\langle r\rangle}\right)=\operatorname{in}_{\succ_{\mathrm{rev}}} \phi_{r}^{-1}\left(I^{\langle r\rangle}\right)+\left(z_{x_{n}^{r}}, \ldots, z_{x_{\ell}^{r}}\right)+\left(z_{m} z_{m^{\prime}}: m m^{\prime} \in\left(x_{n}^{r}, \ldots, x_{\ell}^{r}\right)\right) .
$$

Proof. It is clear that the left-hand side contains the right-hand side. We show that also the reverse inclusion holds. Let

$$
u=z_{m_{1}} z_{m_{2}} \cdots z_{m_{k}} \in \operatorname{in}_{\succ \text { rev }} \phi_{r}^{-1}\left(J^{\langle r\rangle}\right)
$$

be a monomial with $m_{1}>_{\text {rev }} \cdots>_{\text {rev }} m_{k}$. Further assume that $u \notin \operatorname{in}_{\succ_{\text {rev }}} \phi_{r}^{-1}\left(I^{\langle r\rangle}\right)$. We need to show that $u \in\left(z_{x_{n}^{r}}, \ldots, z_{x_{\ell}^{r}}\right)+\left(z_{m} z_{m^{\prime}}: m m^{\prime} \in\left(x_{n}^{r}, \ldots, x_{\ell}^{r}\right)\right)$ in this case.

Since $u \notin \operatorname{in}_{\succ_{\text {rev }}} \phi_{r}^{-1}\left(I^{\langle r\rangle}\right)$, we have $u \notin$ in $_{\succ_{\text {rev }}} \operatorname{ker}\left(\phi_{r}\right)$. Thus, $u$ is standard by Lemma 3.1. We claim $\phi_{r}(u) \in J^{\langle r\rangle}$. Let $f=u+\lambda_{1} v_{1}+\cdots+\lambda_{m} v_{m}+g \in \phi_{r}^{-1}\left(J^{\langle r\rangle}\right)$ be such that $\operatorname{in}_{\succ_{\text {rev }}}(f)=u, g \in \operatorname{ker}\left(\phi_{r}\right), u, v_{1}, \ldots, v_{m}$ are distinct standard monomials and $\lambda_{1}, \ldots, \lambda_{m} \in K$. Then $\phi_{r}(f)=\phi_{r}(u)+\lambda_{1} \phi_{r}\left(v_{1}\right)+\cdots+\lambda_{m} \phi_{r}\left(v_{m}\right) \in J^{\langle r\rangle}$. Since $J$ is a monomial ideal and $\phi_{r}(u), \phi_{r}\left(v_{1}\right), \ldots, \phi_{r}\left(v_{m}\right)$ are distinct monomials, we have $\phi_{r}(u) \in J^{\langle r\rangle}$.

Since, by assumption, $u \notin \phi_{r}^{-1}\left(I^{\langle r\rangle}\right)$, we have

$$
\phi_{r}(u)=m_{1} m_{2} \cdots m_{k} \in\left(x_{n}^{r}, \ldots, x_{\ell}^{r}\right) .
$$

Thus, there is an $\ell \leq i \leq n$ such that $x_{i}^{r}$ divides $\phi_{r}(u)$. If $\operatorname{deg} u=1$, then $u$ must be equal to $z_{x_{i}^{r}}$. If $\operatorname{deg} u>1$, then, by the definition of a standard monomial, there is a $1 \leq j \leq k-1$ such that $x_{i}^{r}$ divides $m_{j} m_{j+1}$. This proves the desired statement.

A monomial ideal $I \subset S$ is called stable if, for any monomial $m \in I$, one has $m\left(x_{i} / x_{\max (m)}\right) \in I$ for any $i<\max (m)$. The following facts are known.

\section{Lemma 3.3.}

(i) For any Cohen-Macaulay standard graded $K$-algebra $A$ with $\operatorname{dim}_{K} A_{1} \leq n$, there is a strongly stable monomial ideal $J \subset S$ such that $S / J$ is CohenMacaulay and $S / J$ has the same Hilbert series as $A$.

(ii) Let $I \subset S$ be a stable monomial ideal such that $S / I$ is a Cohen-Macaulay graded $K$-algebra of dimension $d$. Then $x_{n}, x_{n-1}, \ldots, x_{n-d+1}$ is a linear system of parameters for $S / I$ and $I$ is generated by monomials of degree $\leq$ $\operatorname{deg} h_{S / I}(t)+1$. 
Proof. We only sketch the proof since the statements are well known in commutative algebra. By Macaulay's Theorem on Hilbert functions (see [3, Theorem 4.2.10]) there exists a lexsegment ideal $I$ in a polynomial ring $K\left[x_{1}, \ldots, x_{n-d}\right]$ such that the Hilbert series of $K\left[x_{1}, \ldots, x_{n-d}\right] / I$ equals the $h$-polynomial of $A$. Moreover, the algebra $S / I S$ is a $d$-dimensional Cohen-Macaulay algebra and in particular, it has the same Hilbert series as $A$. Part (i) follows since any lexsegment ideal is stable and it is easy to see that then also $I S$ has to be stable.

Suppose that $I$ is a stable monomial ideal such that $S / I$ is Cohen-Macaulay. A result of Eliahou and Kervaire [7] shows that $I$ is generated by monomials in $K\left[x_{1}, \ldots, x_{n-d}\right]$. This shows that $x_{n}, x_{n-1}, \ldots, x_{n-d+1}$ is a regular sequence of $S / I$ and, therefore, is a l.s.o.p. for $S / I$. Also, since the $h$-polynomial of $S / I$ is equal to the Hilbert series of $S /\left(I+\left(x_{n}, x_{n-1}, \ldots, x_{n-d+1}\right)\right)$, I contains all monomials in $K\left[x_{1}, \ldots, x_{n-d}\right]$ of degree $\operatorname{deg} h_{S / I}(t)+1$. Since $I$ is generated by monomials in $K\left[x_{1}, \ldots, x_{n-d}\right], I$ is generated by monomials of degree $\leq \operatorname{deg} h_{S / I}(t)+1$.

For the proof of Theorem 1.3 we will use the following result for Veronese algebras of the quotient of a stable monomial ideal, which was proven by Eisenbud, Reeves and Totaro [6, Theorem 8].

Lemma 3.4 (Eisenbud-Reeves-Totaro). Let $I \subset S$ be a stable monomial ideal generated by monomials of degree $\leq s$. If $r \geq \frac{s}{2}$, then in $_{\succ_{\mathrm{rev}}} \phi_{r}^{-1}\left(I^{\langle r\rangle}\right)$ is generated by monomials of degree $\leq 2$.

Now we are in the position to prove Theorem 1.3. Recall that a simplicial complex $\Delta$ on $[n]:=\{1,2, \ldots, n\}$ is a collection of subsets of $[n]$, called faces, satisfying that if $F \in \Delta$ and $G \subset F$, then $G \in \Delta$. A simplicial complex is said to be flag if every minimal non-face of $\Delta$ has cardinality $\leq 2$. For a simplicial complex $\Delta$, we write $f_{i}(\Delta)$ for the number of elements $F \in \Delta$ with $|F|=i+1$. The $f$-polynomial of $\Delta$ is the polynomial $f(\Delta, t)=\sum_{i>0} f_{i-1}(\Delta) t^{i}$, where $f_{-1}(\Delta):=1$. The $f$-polynomial of $\Delta$ can be expressed in an algebraic way. Indeed, the $f$-polynomial of a simplicial complex $\Delta$ on $[n]$ is equal to the Hilbert series of $S /\left(\left(x_{F}: F \notin \Delta\right)+\left(x_{1}^{2}, \ldots, x_{n}^{2}\right)\right)$, where $x_{F}:=\prod_{i \in F} x_{i}$. Moreover, $\Delta$ is flag if and only if the ideal $\left(x_{F}: F \notin \Delta\right)+\left(x_{1}^{2}, \ldots, x_{n}^{2}\right)$ is generated by monomials of degree $\leq 2$.

Proof of Theorem 1.3. Part (iii) immediately follows from Theorem 1.1. The unimodality of (ii) is a direct consequence of Theorem 1.2. We prove (i) and the remaining part of (ii).

Fix $r \geq 1$. Since the Hilbert series of $A^{\langle r\rangle}$ only depends on $r$ and the Hilbert series of $A$, by Lemma 3.3 (i), we may assume that $A=S / I$, where $I$ is a stable monomial ideal. Let $\Theta=x_{n}, x_{n-1}, \ldots, x_{n-d+1}$ and $J=I+\left(x_{n}^{r}, \ldots, x_{n-d+1}^{r}\right)$. Then, by Lemma 3.3 (ii), $\Theta$ is a l.s.o.p. for $A=S / I$ and

$$
A_{\Theta}^{\langle r\rangle}=S^{\langle r\rangle} / J^{\langle r\rangle} \cong T_{\langle r\rangle} / \phi_{r}^{-1}\left(J^{\langle r\rangle}\right) .
$$

We now prove (i). Suppose $r \geq \frac{1}{2}\left(\operatorname{deg} h_{A}(t)+1\right)$. Let $\Delta$ be the set of monomials in $T_{\langle r\rangle}$, which are not contained in $\operatorname{in}_{\succ_{\text {rev }}}\left(\phi_{r}^{-1}\left(J^{\langle r\rangle}\right)\right)$. By Lemma 3.3 (ii), $I$ is generated by monomials of degree $\leq \operatorname{deg} h_{A}(t)+1$. Then Lemma 3.2 and Lemma 3.4 say that in $\succ_{\text {rev }}\left(\phi_{r}^{-1}\left(J^{\langle r\rangle}\right)\right)$ is generated by monomials of degree $\leq 2$. This fact shows that $\operatorname{in}_{\succ \text { rev }}\left(\phi_{r}^{-1}\left(J^{\langle r\rangle}\right)\right)$ contains $z_{m}^{2}$ for any variable $z_{m}$ of $T_{\langle r\rangle}$ since $T_{\langle r\rangle} / \phi_{r}^{-1}\left(J^{\langle r\rangle}\right)$ is 
Artinian. This implies that $\Delta$ is a set of square-free monomials. Thus, we may regard $\Delta$ as a simplicial complex. Moreover, since

$$
\text { in }_{\succ_{\text {rev }}}\left(\phi_{r}^{-1}\left(J^{\langle r\rangle}\right)\right)=\left(u: u \text { is a monomial in } T_{\langle r\rangle} \text { with } u \notin \Delta\right)
$$

is generated by monomials of degree $\leq 2, \Delta$ is a flag simplicial complex. Also, by the construction of $\Delta$, we have

$$
f(\Delta, t)=\operatorname{Hilb}\left(T_{\langle r\rangle} / \phi_{r}^{-1}\left(J^{\langle r\rangle}\right), t\right)=\operatorname{Hilb}\left(A_{\Theta}^{\langle r\rangle}, t\right)=h_{A^{\langle r\rangle}}(t),
$$

which proves (i).

Finally, we prove the second part of (ii). Let $\lambda=\operatorname{deg} h_{A\langle r\rangle}(t)$. Suppose $r \geq$ $\operatorname{deg} h_{A}(t)$. Note that in this case it follows from (2.4) that $\lambda \leq d$. By Theorem 1.2, the proof of Theorem 2.2 and Remark 2.3 concerning the location of the gap, there is a linear form $w \in\left(S^{\langle r\rangle}\right)_{1}=S_{r}$ such that

$$
g_{A\langle r\rangle}(t)=\sum_{i=0}^{\left\lfloor\frac{\lambda}{2}\right\rfloor}\left(\operatorname{dim}_{K}\left(A_{\Theta}^{\langle r\rangle} / w A_{\Theta}^{\langle r\rangle}\right)_{i}\right) t^{i} .
$$

Observe

$$
A_{\Theta}^{\langle r\rangle} / w A_{\Theta}^{\langle r\rangle} \cong T_{\langle r\rangle} / \phi_{r}^{-1}\left(J^{\langle r\rangle}+(w)^{\langle r\rangle}\right)
$$

Let $\Gamma$ be the set of monomials of degree $\leq\left\lfloor\frac{\lambda}{2}\right\rfloor$ which are not in $\operatorname{in}_{\succ_{\text {rev }}} \phi_{r}^{-1}\left(J^{\langle r\rangle}+\right.$ $\left.(w)^{\langle r\rangle}\right)$. As we have already seen in the proof of $(\mathrm{i}), \operatorname{in}_{\succ_{\mathrm{rev}}} \phi_{r}^{-1}\left(J^{\langle r\rangle}\right)$ contains $z_{m}^{2}$ for any variable $z_{m}$ of $T_{\langle r\rangle}$. Thus $\Gamma$ can be regarded as a simplicial complex. Then, (3.1) and (3.2) say that $g_{A\langle r\rangle}(t)$ is equal to the $f$-polynomial of $\Gamma$, as desired.

\section{Acknowledgments}

We thank Eran Nevo for helpful comments and suggestions concerning an earlier version of this paper. We are also grateful to two anonymous referees for helpful comments. The first author carried out this work while being a member of the combinatorics group at University of Vienna supported through the FWF-grant no. Y463-N13 of Ilse Fischer. Research of the second author was supported by KAKENHI 22740018.

\section{References}

[1] M. Beck and A. Stapledon, On the log-concavity of Hilbert series of Veronese subrings and Ehrhart series, Math. Z. 264 (2010), 195-207.

[2] F. Brenti and V. Welker, The Veronese construction for formal power series and graded algebras, Adv. Appl. Math. 42 (2009), 545-556.

[3] W. Bruns and J. Herzog, Cohen-Macaulay rings, Cambridge studies in advanced mathematics, Rev. ed., Vol. 39, Cambridge University Press, 1998.

[4] H. Campbell, A. Geramita, I. Hughes, G. Smith and D. Wehlau, Some remarks on Hilbert functions of Veronese algebras, Comm. Algebra 28(3) (2000), 1487-1496.

[5] D. Cox, J. Little and D. O'Shea, Ideals, varieties, and algorithms: an introduction to computational algebraic geometry and commutative Algebra, Springer-Verlag, 1997.

[6] D. Eisenbud, A. Reeves and B. Totaro, Initial ideals, Veronese subrings, and rates of algebras, Adv. Math. 109(2) (1994), 168-187.

[7] S. Eliahou and M. Kervaire, Minimal resolutions of some monomial ideals, J. Algebra 129 (1990), 1-25.

[8] S. Goto and K. Watanabe, On graded rings, I, J. Math. Soc. Japan 30 (1978), 179-213. 
[9] M. Kubitzke and E. Nevo, The Lefschetz property for barycentric subdivisions of shellable complexes, Trans. Amer. Math. Soc. 361(11) (2009), 6151-6163.

[10] M. Kubitzke and V. Welker, Enumerative $g$-theorems for the Veronese construction for formal power series and graded algebras, 2011, http://arxiv.org/abs/1108.2852.

[11] R. Stanley, Weyl groups, the hard Lefschetz theorem, and the Sperner property, SIAM J. Algebraic Discrete Methods 1 (1980), 168-184.

[12] - Combinatorics and Commutative Algebra, in Progress in Mathematics, 2nd ed., Vol. 41, Birkhäuser Boston Inc., Boston, MA, 1996.

[13] B. Sturmfels, Gröbner bases and convex polytopes, University Lecture Series, Vol. 8, American Mathematical Society, Providence, RI, 1996.

[14] J. Watanabe, The Dilworth number of Artinian rings and finite posets with rank function, Commutative algebra and combinatorics (Kyoto, 1985), Vol. 11 of Adv. Stud. Pure Math., 303312, North-Holland, Amsterdam, 1987.

FB 12 - Institut für Mathematik, Goethe-Universität, Robert-Mayer Strasse 10, D-60325 Frankfurt am Main, Germany

E-mail address: kubitzke@math.uni-frankfurt.de

Department of Mathematical Science, Faculty of Science, Yamaguchi University, 1677-1 Yoshida, Yamaguchi 753-8512, JAPAN.

E-mail address: murai@yamaguchi-u.ac.jp 
